\title{
Assessment of Vibrational Non-Equilibrium Effect on Detonation Cell Size
}

\author{
Lisong Shi, Hua Shen, Peng Zhang, Deliang Zhang \& Chihyung Wen
}

To cite this article: Lisong Shi, Hua Shen, Peng Zhang, Deliang Zhang \& Chihyung Wen (2017) Assessment of Vibrational Non-Equilibrium Effect on Detonation Cell Size, Combustion Science and Technology, 189:5, 841-853, DOI: 10.1080/00102202.2016.1260561

To link to this article: http://dx.doi.org/10.1080/00102202.2016.1260561

Accepted author version posted online: 17

Nov 2016.

Published online: 17 Nov 2016.

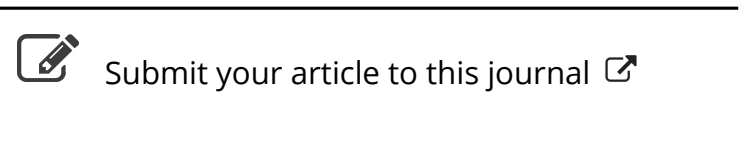

Џ Article views: 219

Q View related articles $\square$

View Crossmark data ऍ 


\title{
Assessment of Vibrational Non-Equilibrium Effect on Detonation Cell Size
}

\author{
Lisong Shi ${ }^{a}$, Hua Shen ${ }^{a}$, Peng Zhanga ${ }^{a}$ Deliang Zhang ${ }^{b}$, and Chihyung Wen ${ }^{a}$ \\ aDepartment of Mechanical Engineering, The Hong Kong Polytechnic University, Kowloon, Hong Kong; bState \\ Key Laboratory of High Temperature Gas Dynamics, Institute of Mechanics, Chinese Academy of Sciences, \\ Beijing, China
}

\begin{abstract}
To resolve the discrepancy between the numerical detonation cell size and experimental observations, simulations are conducted with a detailed thermochemical reaction model for a premixed argondiluted hydrogen-oxygen mixture. Four different scenarios are considered: (i) The whole system is in thermodynamic equilibrium; (ii) the vibrational relaxation is considered and the translational-rotational temperature is used as the dominant temperature of the chemical reactions; (iii) the same non-equilibrium effect as in the second scenario is used along with Park's two-temperature model to account for the effect of vibrational temperature on chemical reaction rates; and (iv) a more physically consistent vibration-chemistry-vibration coupling model is adopted. The simulated detonation cell widths for the first and second scenarios are significantly lower than the experimental measurements, whereas reasonable agreement is observed for the third and fourth scenarios. These results confirm that the involvement of vibrational relaxation in the chemical reactions is an important mechanism in gaseous detonation.
\end{abstract}

\section{ARTICLE HISTORY}

Received 12 August 2016

Revised 16 October 2016

Accepted 7 November 2016

\section{KEYWORDS}

Cellular structure; Coupledvibration-chemistryvibration (CVCV) model; Detonation; Twotemperature model; Vibrational non-equilibrium

\section{Introduction}

Extensive numerical studies have been conducted on the investigation of detonation cellular structures. Detailed chemical reaction models have been adopted in an effort to reveal the physics behind detonations ( $\mathrm{Hu}$ et al., 2005; Oran et al., 1998). Recent numerical studies have been confronted with the difficulty of matching the simulated cell size with the experimental observations. For a stoichiometric argon-diluted hydrogen-oxygen mixture, the cells are approximately half the measured values, which is attributed to neglect of one of the basic physical processes under supersonic reactive flow, i.e., the vibrational relaxation process, which is neglected when calibrating detailed chemical reaction models (Taylor et al., 2013a).

Within the conditions of detonation, the ratio of ignition delay time over the characteristic timescale for different molecules to reach vibrational equilibrium ranges from 1 to an order of $10^{4}$ according to the estimations of Taylor et al. (2013b). For reference, the ratio of the ignition delay time over the vibrational relation time under the post-shock state of approximately 28 atm

CONTACT Chihyung Wen chihyung.wen@polyu.edu.hk $\Theta$ Department of Mechanical Engineering, The Hong Kong Polytechnic University, Kowloon, Hong Kong.

This work has been presented in Work in Progress Poster (WiPP), 36th International Symposium on Combustion, COEX, Seoul, Korea July 31-August 5, 2016.

Color versions of one or more of the figures in the article can be found online at www.tandfonline.com/gcst. 
and $1540 \mathrm{~K}$ for a Chapman-Jouguet detonation in stoichiometric $\mathrm{H}_{2}$-air at 1 atm and $300 \mathrm{~K}$, assuming that the gas is at thermodynamic equilibrium, is less than 3 for $\mathrm{H}_{2}$ and less than 2 for $\mathrm{N}_{2}$. Within the induction zone after the shock compression, oxygen is not always fully excited vibrationally, and the averaged reaction rate is thus lower than that in the thermal equilibrium assumption (Schott and Kinsey, 1958). Therefore, the important physical mechanism of vibrational non-equilibrium may be important in detailed chemical reaction mechanisms when applied to the simulation of detonation or, more generally, reactive high-speed flows. Unfortunately, no currently available simulation studies have been conducted to quantitatively study the effect on cell size of vibrational relaxation and coupling between vibrational nonequilibrium and chemical reactions.

In this article, both one-dimensional (1D) and two-dimensional (2D) numerical simulations of unsteady detonation waves were performed using a recently developed detailed chemical mechanism for high-pressure combustion of hydrogen. Quantitative comparisons of halfreaction thickness and cell widths from thermodynamic equilibrium cases to thermodynamic non-equilibrium cases are discussed in detail to examine the effect of vibrational non-equilibrium. The interactions between vibrational non-equilibrium and chemical reactions are treated with Park's two-temperature model (1989) and the more recent coupled-vibration-chemistryvibration (CVCV) model (Knab et al., 1995; Koo et al., 2015; Voelkel et al., 2015).

\section{Physical models and methods}

The shock-capturing space-time conservation element and solution element (CE/SE) method, originally proposed by Chang (1995), has been extensively developed and used to handle shock waves (Shen and Wen, 2016; Shen et al., 2015a, 2015b), detonation waves (Shen et al., 2011), and hypersonic non-equilibrium flows (Shen et al., 2014). In this study, the secondorder CE/SE scheme is used to solve the reactive Euler equations. The stiff source terms are explicitly integrated as ordinary differential equations in an operator-split manner (Oran and Boris, 2005; Ropp and Shadid, 2009), which is employed to decouple hydrodynamic transport and chemical reactions. The advection and reaction terms are separated into a set of singleterm equations, i.e., the continuity Eq. (1):

$$
\frac{\partial \rho_{i}}{\partial t}+\nabla \cdot \rho_{i} \mathrm{U}=\omega_{i}
$$

is treated as:

$$
\begin{gathered}
\frac{\partial \rho_{i}}{\partial t}=-\nabla \cdot \rho_{i} \mathrm{U} \\
\frac{\partial \rho_{i}}{\partial t}=\omega_{i}
\end{gathered}
$$

where $\rho_{i}$ is the species density, $\mathbf{U}$ the velocity, and $\omega_{i}$ the chemical reaction source term of species $i$. Thus, the calculation constitutes two steps: advection step (Eq. (2)) and reaction step (Eq. (3)). If the discrete solution operators of Eqs. (2) and (3) are denoted as $H$ and $R$, respectively, the entire splitting scheme becomes:

$$
\rho_{i}^{n+1}=R H \rho_{i}^{n}
$$


In the reaction step, the sub-cycling is used, i.e., several smaller time steps within one global time step, to resolve all the time scales of the chemical reactions.

We use the recently developed detailed chemical kinetics and thermodynamic data of Burke et al. (2012) for high-pressure hydrogen combustion, which have been endorsed for detonation simulations by other researchers (Taylor et al., 2013a). The NASA Glenn coefficients (McBride et al., 2002) are used to evaluate the chemical equilibrium constants required for the backward reaction rates. The values of specific heat at constant volume are presented by the following equation:

$$
C_{v}=C_{v, \mathrm{tr}}+C_{v, \mathrm{v}}
$$

The total energy per unit volume of a mixture is given by:

$$
E=\sum_{i=1}^{N s} \rho_{i} h_{i}^{f}+\sum_{i=1}^{N s} \rho_{i} C_{v, \mathrm{tr}, i} T_{\mathrm{tr}}+\frac{1}{2} \rho\left(u^{2}+v^{2}\right)+\sum_{i=\text { molecule }} \rho_{i} \cdot R_{i} \cdot \frac{\theta_{\mathrm{v}, i}}{\exp \left(\theta_{\mathrm{v}, i} / T_{\mathrm{v}}\right)-1}
$$

in which the last term represents the vibrational energy of the mixture. The characteristic vibrational temperatures of different species are listed in Table 1. Note that the vibrational energy of $\mathrm{H}_{2} \mathrm{O}_{2}$ is neglected due to its extremely low mass fraction (on the order of $10^{-5}$ or less). Notably, after shock compression, translational equilibrium is rapidly established, typically in less than 10 molecular collisions. Reaching rotational equilibrium for most small molecules typically requires 10 to 20 molecular collisions, but reaching vibrational equilibrium requires thousands of collisions (Taylor et al., 2013a). Therefore, we only consider here the non-equilibrium effects between vibrational energy and translationalrotational energy. The energy exchange rate is determined by the Landau-Teller relaxation model (Gnoffo et al., 1989) as:

$$
S_{\mathrm{tr}-\mathrm{v}}=\frac{\rho C_{v, \mathrm{v}}}{\tau}\left(T_{\mathrm{tr}}-T_{\mathrm{v}}\right)
$$

Based on different assumptions, the following four physical models are tested in this article.

- Model (i): Vibrational energy is assumed to be in equilibrium with translationalrotational energy, i.e., $T_{\mathrm{v}}=T_{\mathrm{tr}}$ in Eq. (6). The governing equations are the conventional reactive Euler equations.

- Model (ii): Considering the vibrational relaxation, an additional transport equation is used to describe the evolution of vibrational energy. The non-equilibrium effect results in different values of $T_{\mathrm{tr}}$ and $T_{\mathrm{v}}$. The choice of the dominant temperature of

Table 1. Characteristic vibrational temperature of molecules.

\begin{tabular}{lc}
\hline Molecule & $\theta_{\mathrm{v}}(\mathrm{K})$ \\
\hline $\mathrm{H}_{2}$ & 5989 \\
$\mathrm{O}_{2}$ & 2250 \\
$\mathrm{OH}$ & 5140 \\
$\mathrm{HO}_{2}$ & $1577,2059,5325$ \\
$\mathrm{H}_{2} \mathrm{O}$ & $2297,5266,5409$ \\
\hline
\end{tabular}


the chemical reaction rate is crucial. In this model, we neglect vibrational-chemical coupling and use $T_{\mathrm{tr}}$ as the dominant temperature for all reactions.

- Model (iii): Except for the choice of dominant temperature, all other settings remain consistent with model (ii). Considering the vibrational relaxation as in model (ii), Park's two-temperature model (Park, 1989) is used in which the influence of the vibrational temperature on the chemical reaction rates is accounted for. Evidently, the concept of the Arrhenius equation is that two molecules will react when the mutual energy exceeds a certain minimum value. It is reasonable to assume that the vibrational cold mixture is less likely to react than that of the mixture that is fully excited vibrationally. Although it may be ambiguous to ascertain the exact contribution of $T_{\mathrm{v}}$, an average temperature $T_{\text {avg }}$, defined by the square root of the product of $T_{\text {tr }}$ and $T_{\mathrm{v}}$, is widely used as the dominant temperature of the Arrhenius reaction rate in the community of hypersonic non-equilibrium flows (Park, 1989; Shen et al., 2014). Here, we try to use this concept to investigate the effect of vibrational nonequilibrium on the high-speed reactive flows.

- Model (iv): Another approach to examine the effect of vibrational non-equilibrium is the physically consistent CVCV model, which is derived on the assumptions of the $T_{\mathrm{v}}$-Boltzmann populated vibrational energy mode and truncated harmonic oscillator (Knab et al., 1995). In this model, the chemical reaction rate is modified by an efficiency function, $\varphi\left(T_{\mathrm{tr}}, T_{\mathrm{v}}\right)$ :

$$
k\left(T_{\mathrm{tr}}, T_{\mathrm{v}}\right)=\varphi\left(T_{\mathrm{tr}}, T_{\mathrm{v}}\right) k_{\mathrm{eq}}\left(T_{\mathrm{tr}}\right)
$$

The efficiency function is calculated as:

$$
\varphi\left(T_{\mathrm{tr}}, T_{\mathrm{v}}\right)=\frac{Q\left(T_{\mathrm{tr}} ; E_{\mathrm{d}}\right)}{Q\left(T_{\mathrm{v}} ; E_{\mathrm{d}}\right)} \cdot \frac{e^{-\alpha E_{\mathrm{a}} / R T} Q\left(\Gamma ; \alpha E_{\mathrm{a}}\right)+Q\left(T^{0} ; E_{\mathrm{d}}\right)-Q\left(T^{0} ; \alpha E_{\mathrm{a}}\right)}{e^{-\alpha E_{\mathrm{a}} / R T} Q\left(-U ; \alpha E_{\mathrm{a}}\right)+Q\left(T^{*} ; E_{\mathrm{d}}\right)-Q\left(T^{*} ; \alpha E_{\mathrm{a}}\right)}
$$

where $\frac{1}{\Gamma}=\frac{1}{T_{\mathrm{v}}}-\frac{1}{T_{\mathrm{tr}}}-\frac{1}{U}, \frac{1}{T^{0}}=\frac{1}{T_{\mathrm{v}}}-\frac{1}{U}, \frac{1}{T^{*}}=\frac{1}{T_{\mathrm{tr}}}-\frac{1}{U}$. The coefficient $a$, which determines the fraction of required energy contributed by the translational energy, is selected as 0.8 , and $U=E_{\mathrm{d}} /(5 R)$ as assessed in Knab et al. (1995), Koo et al. (2015), and Voelkel et al. (2015). $Q(T ; E)=\frac{1-\mathrm{e}^{-E /(R T)}}{1-\mathrm{e}^{-\theta_{\mathrm{V}} / T}}$ denotes the partition function truncated below energy $E$.

\section{Numerical results and discussion}

\section{$1 D$ tests}

The mixture was composed of stoichiometric hydrogen oxygen diluted with $70 \%$ argon at a temperature of $300 \mathrm{~K}$, and the initial pressures were $0.1,0.2,0.3$, and $0.4 \mathrm{~atm}$, respectively, identical to the initial conditions listed in Taylor et al. (2013a). As what will be demonstrated in a later section, the meshes were uniformly distributed with $\Delta x=5 \mu \mathrm{m}$. Figure 1 shows the propagating pressure profiles of the mixture at $0.4 \mathrm{~atm}$ using model (i). The detonation velocity is $1637 \mathrm{~m} / \mathrm{s}$. The detonation is shown as initially overdriven but quickly reaches a self-sustained velocity. Good agreement is observed compared to the Chapman-Jouguet velocity of $1668 \mathrm{~m} / \mathrm{s}$. Insignificant differences in the detonation wave speeds were found among the four different models. Figure 2 shows that the calculated half-reaction thickness by using model (ii) is less than 


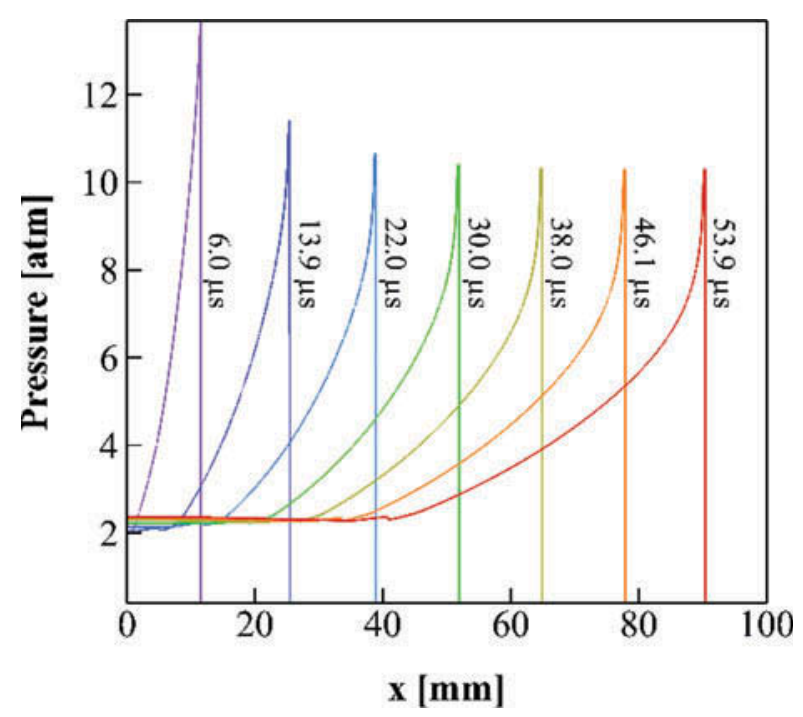

Figure 1. Propagating pressure profiles of model (i) with initial pressure of $0.4 \mathrm{~atm}$ and temperature of $300 \mathrm{~K}$.

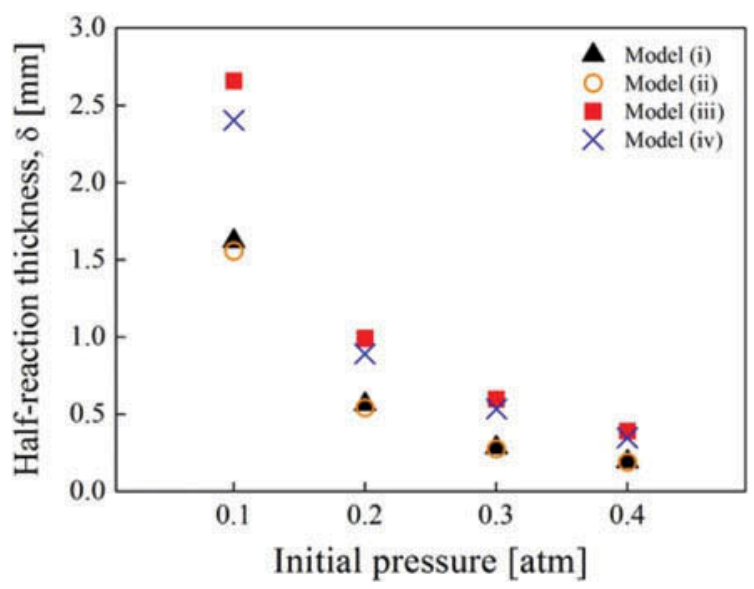

Figure 2. Half-reaction thickness at different initial pressures with an initial temperature of $300 \mathrm{~K}$.

that of model (i) only by an extremely small value. However, the half-reaction thickness is significantly increased by using models (iii) or (iv). The distance between the shock front and the position at which $\mathrm{H}_{2}$ is half consumed is defined as the half-reaction thickness, $\delta$. At $0.1 \mathrm{~atm}$, when the collision frequency is low, the two-temperature model seems to overestimate the vibrational relaxation, as mentioned by Bender et al. (2015) and Panesi et al. (2014). These halfreaction thickness features are further demonstrated in Figure 3.

Figure 3 illustrates the temperature profiles and $\mathrm{H}_{2}$ distribution at $0.1 \mathrm{~atm}$ and $300 \mathrm{~K}$. As shown in Figure 3a, in the simulation using model (i), the mixture experiences an induction period after being compressed to the post-shock conditions. Because it is thermally perfect, the mixture soon undergoes exothermic reactions that increase the $T_{\mathrm{tr}}$. However, instead of the 


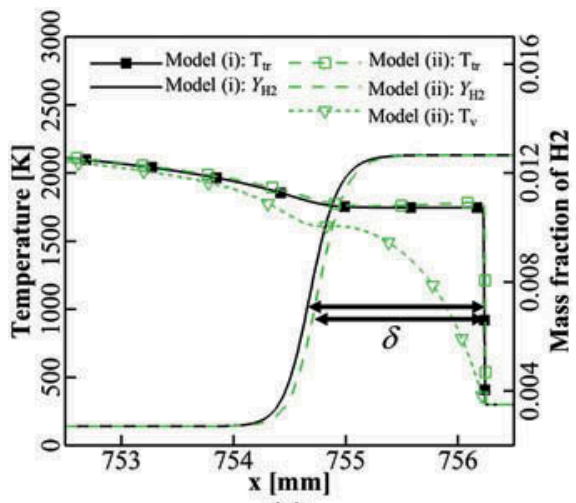

(a)

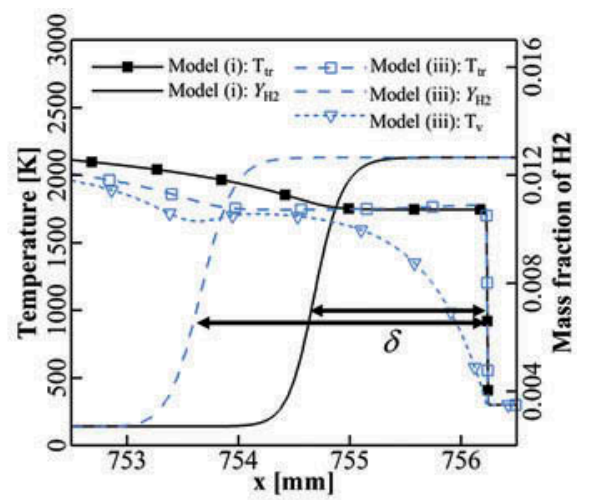

(b)

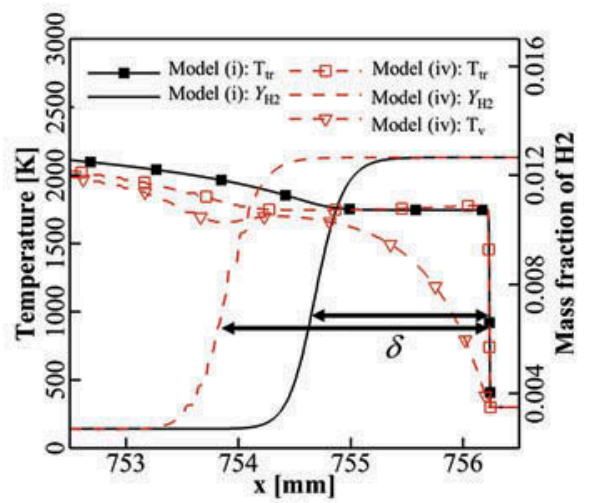

(c)

Figure 3. Temperature and $\mathrm{H}_{2}$ distributions with initial condition of $0.1 \mathrm{~atm}$ and $300 \mathrm{~K}$ : (a) models (i) and (ii), (b) models (i) and (iii), (c) models (i) and (iv). Note that in models (ii), (iii), and (iv), $T_{\text {tr }}$ exhibits overshot behind shock compared to model (i).

infinitely fast transfer rate between translational energy and vibrational energy, in the simulation of model (ii), the molecules are almost vibrational cold behind the shock. The post-shock $T_{\mathrm{tr}}$ is even slightly higher than that in model (i). This could be interpreted from Eq. (6): the relatively slow vibrational relaxation process, which implies less energy in the vibrational mode and more energy in the translational-rotational mode, prompted $T_{\text {tr }}$ to a higher value. However, the minor increase in dominant temperature $T_{\text {tr }}$ accelerates the process of the reaction and somewhat narrows the half-reaction thickness. In Figure $3 \mathrm{~b}$, however, due to the fact that upon shock compression, $T_{\text {tr }}$ undergoes an instantaneous increase, $T_{\mathrm{v}}$ approaches the equilibrium state gradually. As time is required for the molecules to become vibrationally excited before the onset of the severe chemical reaction, the half-reaction thickness in these cases using model (iii) retains an evidently elongated distance (1.64 to 1.71 times that of models (i) and (ii) in these cases). Similar effects can be observed from the case using model (iv), as seen in Figure $3 c$. According to the semi-empirical correlation of the $\lambda / \delta$ ratio with regard to the conditions of the mixture (Gavrikov et al., 2000), the estimation of $1 \mathrm{D}$ detonation $\delta$ can roughly describe the effects of the different models on simulating the detonation cell width. 


\section{D tests}

To begin the $2 \mathrm{D}$ simulations, the computational domain of the $2 \mathrm{D}$ cases was set at $7.76 \mathrm{~mm}$ wide, considering the computational efficiency. The grid independence tests were first conducted. Three different uniform mesh sizes were examined on models (ii) and (iv), with $\Delta h=10.0 \mu \mathrm{m}, 5.0 \mu \mathrm{m}$, and $2.5 \mu \mathrm{m}$, respectively. As shown in Figures 4 and 5 , the averaged cell sizes change dramatically when the mesh sizes decrease from $10 \mu \mathrm{m}$ to $5 \mu \mathrm{m}$; however, further refining the mesh size does not affect the cell size or cell regularity significantly. The grid independence tests were considered convergent at $\Delta h=5.0 \mu \mathrm{m}$. All of the simulations in the current research were conducted using a mesh size of $5.0 \mu \mathrm{m}$ thereafter.

Using the simulation of Taylor et al. (2013a) as the benchmark, the computational domain was filled with stoichiometric $\mathrm{H}_{2}-\mathrm{O}_{2}$ diluted with $70 \% \mathrm{Ar}$ at an initial pressure of $0.4 \mathrm{~atm}$ and initial temperature of $300 \mathrm{~K}$. Adiabatic slip wall boundary conditions were adopted at the upper and lower edges of the simulation domain. The mixture was ignited by a small sinusoidal disturbance region with high enthalpy near the left wall. The cell structure performed immediate changes at the very beginning of detonation and continued to adjust to a relatively steady pattern after propagating in the rightward direction for a certain distance. Figure 6 shows the instant contours of model (i) with steady averaged detonation velocity. The fronts of the detonation waves are modestly corrugated.

It is difficult to measure the half-reaction thickness along the shock front because it varies due to the existence of moving triple points. Nevertheless, it is clear that the values
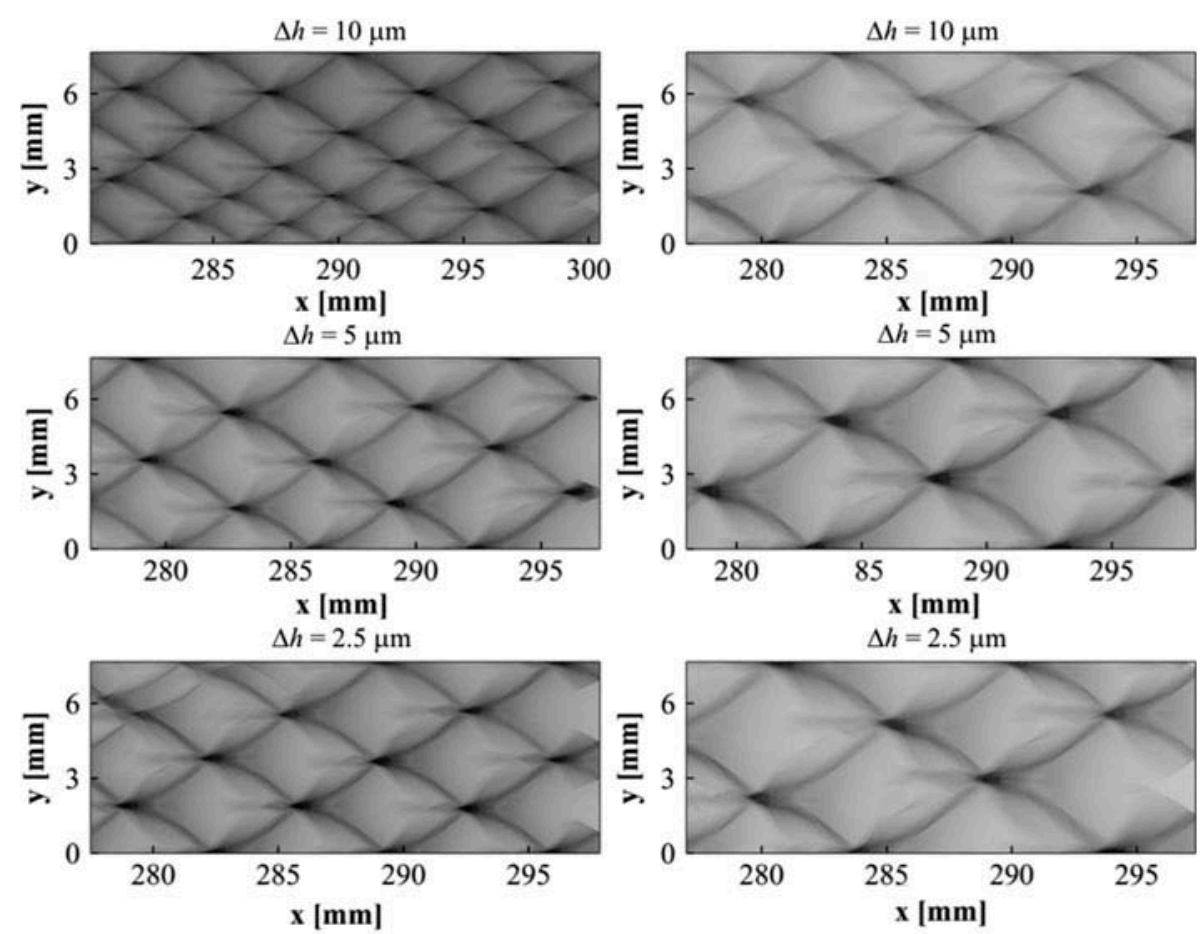

(a) Model (ii)

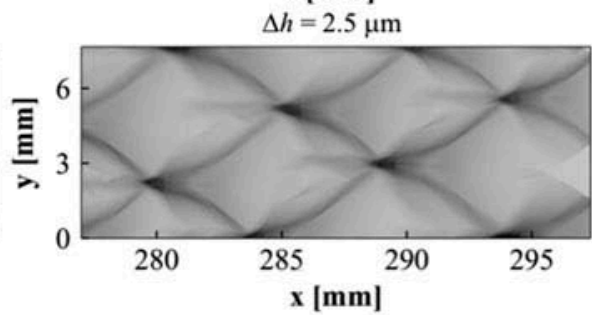

(b) Model (iv)

Figure 4. Numerical soot foils of models (ii) and (iv) using three different mesh sizes. 


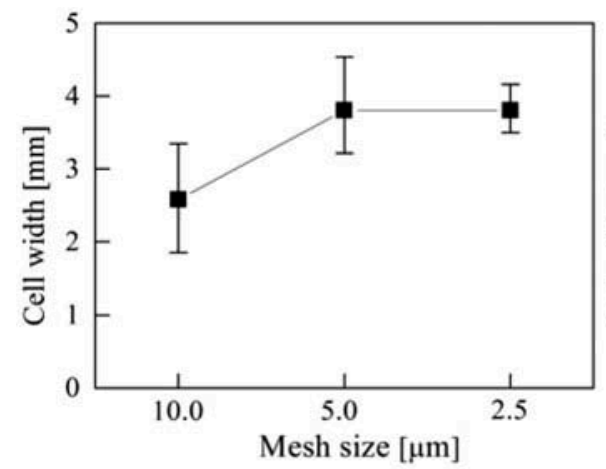

(a) Model (ii)

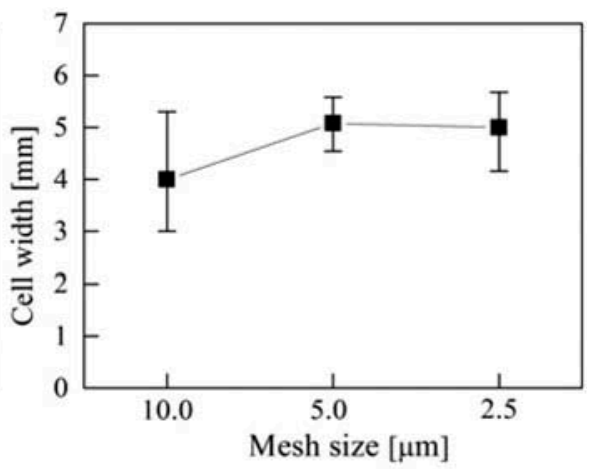

(b) Model (iv)

Figure 5. Influence of the mesh sizes on the detonation cell sizes.

(a) Pressure

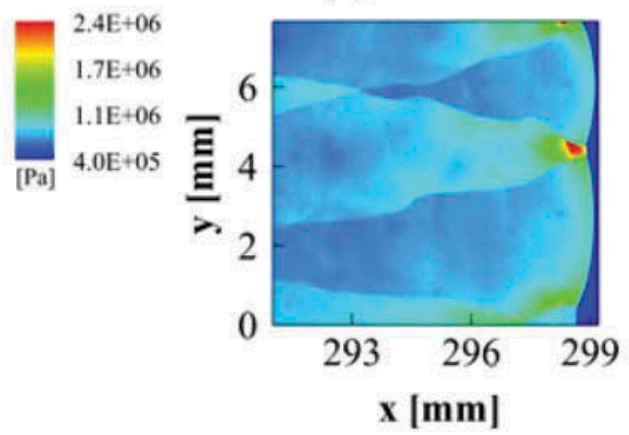

(c) $\mathrm{OH}$ mass fraction

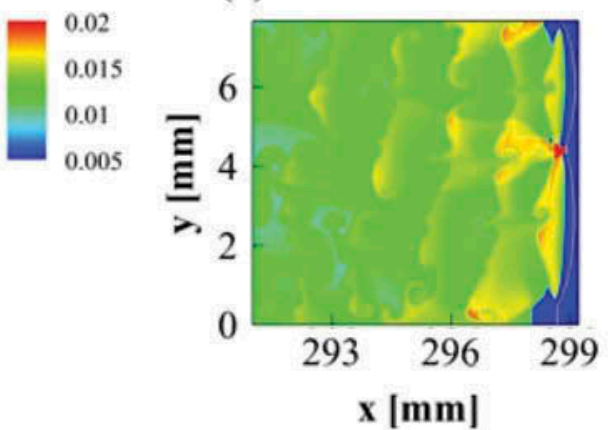

(b) Temperature

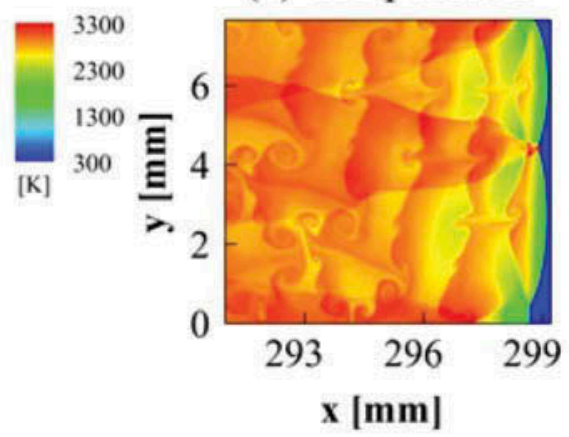

(d) Numerical soot foil

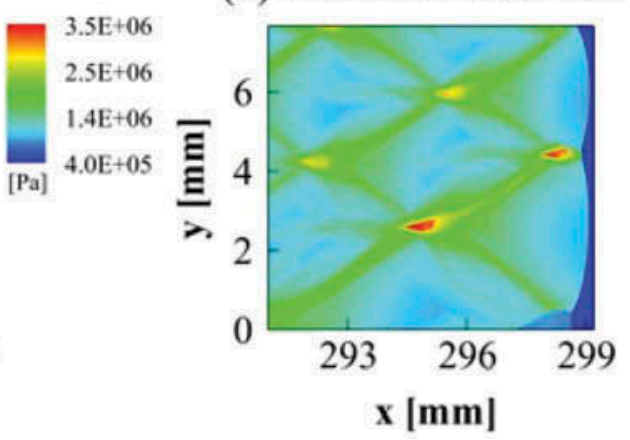

Figure 6. (a) Pressure, (b) temperature, (c) $\mathrm{OH}$ field, and (d) numerical soot foil near front of detonation wave using model (i).

of $\delta$ derived by models (iii) and (iv) are generally much wider than those derived by the other two models (Figure 7).

The numerical soot foil was obtained by recording the maximum pressure of the simulation domain as the detonation wave propagated. Snapshots of the numerical soot foil from the four models are shown in Figure 8. In general, the cell size distribution is 

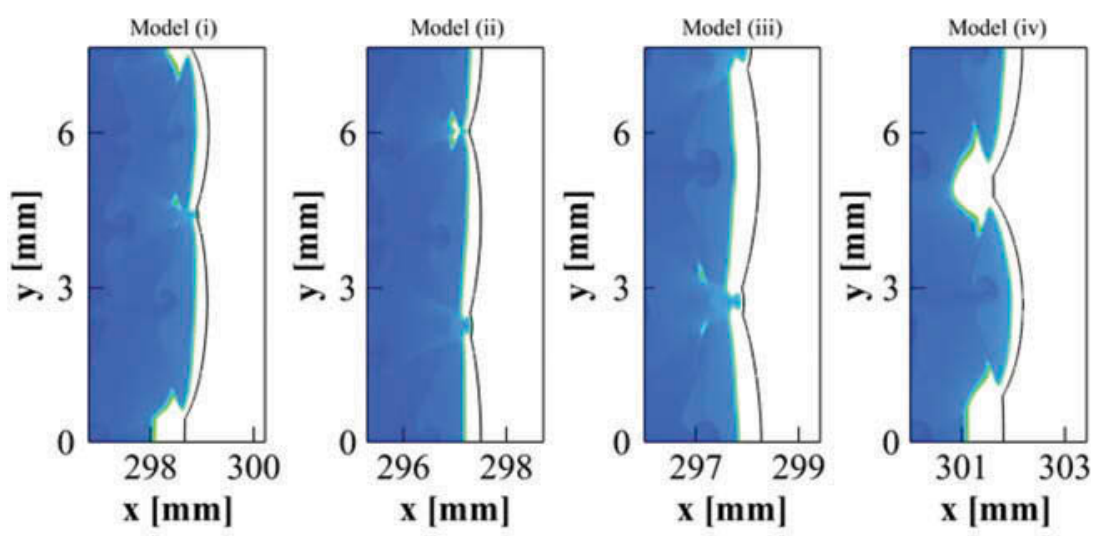

Figure 7. Contour of $\mathrm{H}_{2}$ as magnified near detonation front; front is indicated by solid black line.

(a)

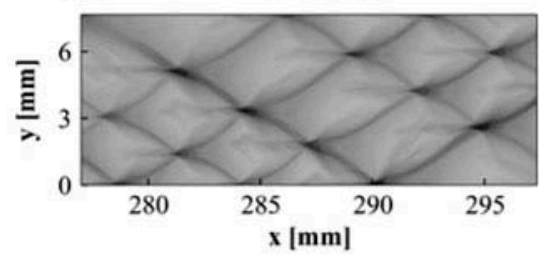

(c)

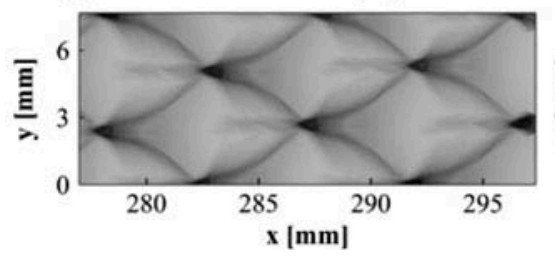

(b) Model (ii)

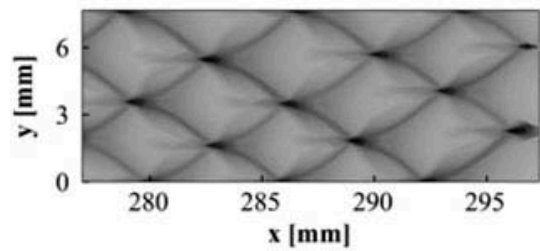

(d)

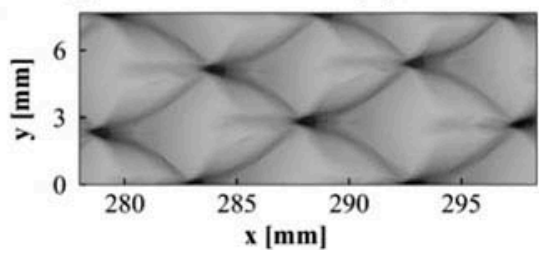

Figure 8. Numerical soot foils using four models.

fairly regular in all four models, which is similar to the findings of Taylor et al. (2013a). The cell structure regularity and averaged cell size in Figure 8a are very similar to those in Figure $8 \mathrm{~b}$. The cells in Figures $8 \mathrm{c}$ and $8 \mathrm{~d}$, however, are markedly enlarged. These observations correspond to the expectations from the findings of the $1 \mathrm{D}$ calculations in the previous section.

Figure 9 shows the average width of the detonation cells within the simulation domain. The computed detonation cell widths in the first scenario range from $2.74 \mathrm{~mm}$ to $5.10 \mathrm{~mm}$ and $3.22 \mathrm{~mm}$ to $4.54 \mathrm{~mm}$ in the second scenario. In the third scenario, the computed cell widths dramatically increase to $4.54 \mathrm{~mm}$ to $5.30 \mathrm{~mm}$, whereas they increase to $4.54 \mathrm{~mm}$ to $5.58 \mathrm{~mm}$ in the fourth scenario. It is noted that the average cell width of model (ii) is similar to that of model (i), whereas the average cell widths in models (iii) and (iv) are 1.32 and 1.34 times the average value of those in model (i), respectively. The average cell widths of models (i) and (ii) are consistent with the numerical simulation results of Taylor et al. (2013a) and are significantly lower than the experimental measurement by a factor of 


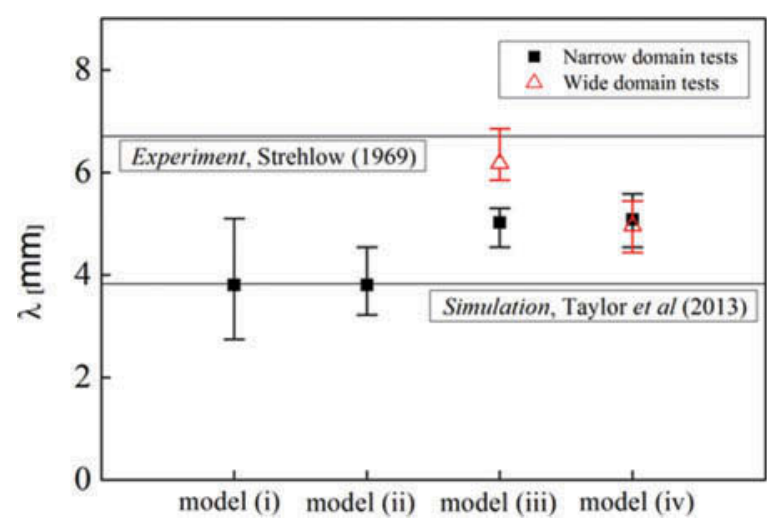

Figure 9. Comparison of detonation cell width obtained in four models.

approximately 2 for this low-pressure, dilute mixture, despite the use of different fluid solvers and the same kinetic model. The general agreement of the results suggests that the assumption of thermodynamic equilibrium may not apply over a large portion of the reactive flow in a detonation. Compared to the cell size measured in the experiment (Strehlow and Engel, 1969), the disparity in cell width has been greatly narrowed down to a factor of 1.33 and 1.32 for models (iii) and (iv), respectively.

Because the values of $C_{v}$ are approximated only by the summation of $C_{v, \text { tr }}$ and $C_{v, v}$ (Eq. (5)), this approach precludes the contribution of the dissociation to the thermodynamic value. This is unavoidable when illustrating the influence of the vibrational relaxation without other distractions. Although this might undoubtedly induce uncertainties into the simulation, such as the lack of accuracy for lateral comparison with previous work in which experimental fitting thermodynamics were adopted, this approach extracts the bias induced by the inconsistency of thermodynamic expression in the governing equations of the different models. Besides the vibrational non-equilibrium, other factors, including mode-locking caused by the limitation of the simulation domain, boundary heat loss, and $3 \mathrm{D}$ effects, may also considerably affect the detonation cell width. Even with these uncertainties, the apparent distinction between the four models already indicates that vibrational relaxation can be the possible reason for the difference between previous numerical studies and the present experimental measurements.

\section{Mode-locking tests}

Considering possible mode-locking effects when the channel width is close to the detonation cell width, two more simulations using Park's two-temperature model and the CVCV model were conducted with the computational domain width increased from $7.76 \mathrm{~mm}$ to $25.0 \mathrm{~mm}$. Figure 10 presents the numerical soot foils for models (iii) and (iv) using the large simulation domain width. The result indicates that the simulation domain size can affect the cell size to a certain extent. As shown in Figure 9, when a large computational width is used, the average cell size for model (iv) changes insignificantly, whereas that for model (iii) increases from $75 \%$ to $92 \%$ of the experimental value. Considering the uncertainties in the experiment, reasonable agreement between the simulations of models (iii) and (iv) and the experiment is observed. 

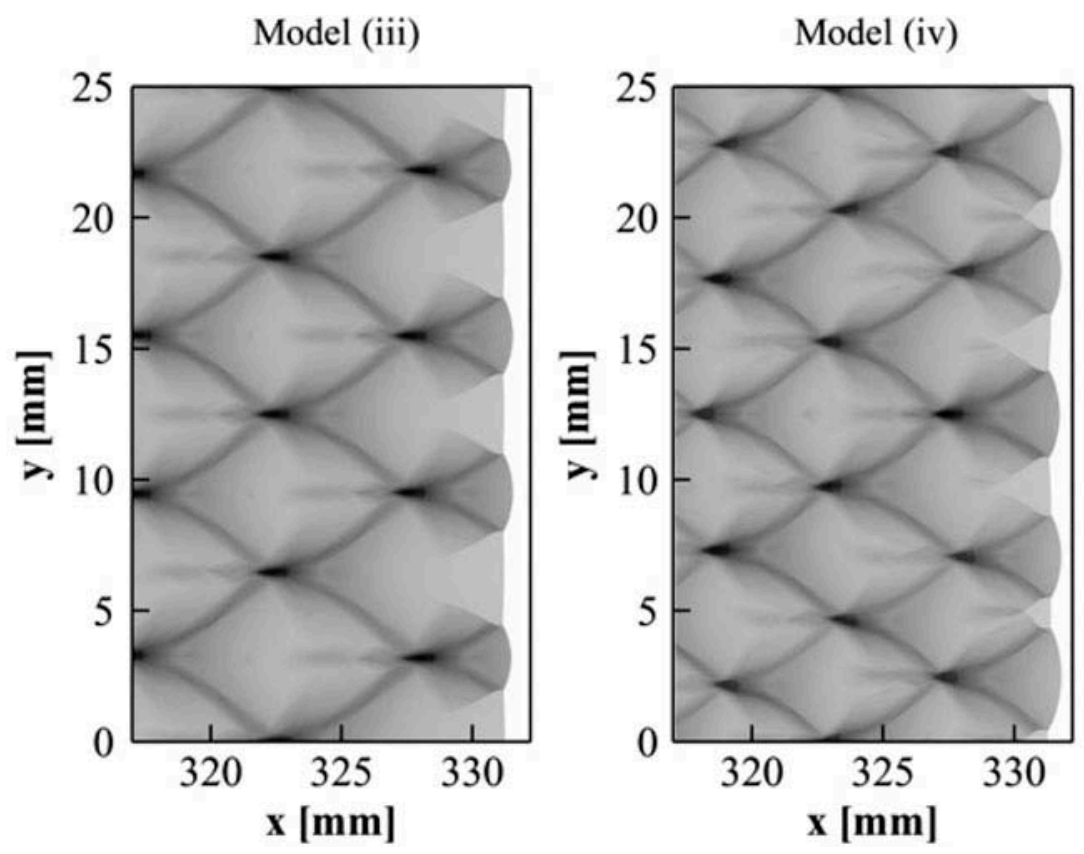

Figure 10. Mode-locking tests for models (iii) and (iv).

\section{Conclusions}

The exclusive involvement of vibrational relaxation in the simulation has insignificant influence on the disagreement of detonation cell width with the experimental result if the dominant temperature of the chemical reaction was maintained at $T_{\mathrm{tr}}$. Commensurate with the nature of the assumption that the reaction occurs in extremely combustible conditions, the half-reaction thickness behind the shock could be affected by vibrational non-equilibrium and vibrational relaxation of the gas molecules that occurs on time scales similar to the ignition delay times for the detonations. The vibrational cold state of the molecules might leverage the reaction rates. The reasonable agreement found between the numerically simulated cell width using the geometrically averaged temperature in Park's two-temperature/efficiency function in the CVCV model and the experimental measurement verified the necessity of involving the contribution of the vibrational relaxation process in the evaluation of chemical reaction rates, a process that is presently ignored when calibrating detailed chemical reaction models. Furthermore, this article reveals the physical mechanism of the effect of vibrational relaxation on the detonation cell size. The contribution from vibrational temperature could vary within a certain range, which will require further careful investigation.

\section{Funding}

This research was supported by the opening project of State Key Laboratory of Explosion Science and Technology (Beijing Institute of Technology; Project No. KFJJ15-09M), and the opening project of Key Laboratory of High Temperature Gas Dynamics (Institute of Mechanics, Chinese Academy of Sciences; Project No. LHD2016KF05). The authors also would like to thank the financial support by the Natural Science Foundation of China (Contract No. 11372265). 


\section{Nomenclature}

$C_{v} \quad$ specific heat at constant volume

$C_{v, \text { tr }}$ translational-rotational specific heat at constant volume

$C_{v, \mathrm{v}} \quad$ vibrational specific heat at constant volume

$E_{\mathrm{a}} \quad$ activation energy

$E_{\mathrm{d}} \quad$ dissociation energy

$h^{\mathrm{f}} \quad$ enthalpy of formation

$k_{\text {eq }}$ reaction rate at vibrational equilibrium condition

$R$ gas constant

$S_{\text {tr-v }}$ energy exchange rate between translational-rotational and vibrational energy

$T_{\text {tr }} \quad$ translational-rotational temperature

$T_{\mathrm{v}} \quad$ vibrational temperature

$u \quad$ velocity along $x$-direction

$v \quad$ velocity along $y$-direction

$Y_{\mathrm{H} 2}$ mass fraction of $\mathrm{H}_{2}$

\section{Greek symbols}

$\delta$ half-reaction thickness

$\lambda$ detonation cell width

$\rho$ density

$\tau$ averaged Landau-Teller relaxation time

$\theta_{\mathrm{v}} \quad$ characteristic vibrational temperature

\section{References}

Bender, J.D., Valentini, P., Nompelis, I., Paukku, Y., Varga, Z., Truhlar, D.G., Schwartzentruber, T., and Candler, G.V. 2015. An improved potential energy surface and multi-temperature quasiclassical trajectory calculations of N2+ N2 dissociation reactions. J. Chem. Phys., 143(5), 054304.

Burke, M.P., Chaos, M., Ju, Y., Dryer, F.L., and Klippenstein, S.J. 2012. Comprehensive $\mathrm{H}_{2} / \mathrm{O}_{2}$ kinetic model for high-pressure combustion. Int. J. Chem. Kinet., 44(7), 444-474.

Chang, S.C. 1995. The method of space-time conservation element and solution element-A new approach for solving the Navier-Stokes and Euler equations. J. Comput. Phys., 119(2), 295-324.

Gavrikov, A.I., Efimenko, A.A., and Dorofeev, S.B. 2000. A model for detonation cell size prediction from chemical kinetics. Combust. Flame, 120(1), 19-33.

Gnoffo, P.A., Gupta, R.N., and Shinn, J.L. 1989. Conservation equations and physical models for hypersonic air flows in thermal and chemical nonequilibrium.NASA TP-2867. NASA Langley, Hampton, VA.

Hu, X.Y., Zhang, D.L., Khoo, B.C., and Jiang, Z.L. 2005. The structure and evolution of a twodimensional $\mathrm{H}_{2} / \mathrm{O}_{2} /$ Ar cellular detonation. Shock Waves, 14(1-2), 37-44.

Knab, O., Frühauf, H.H., and Messerschmid, E.W. 1995. Theory and validation of the physically consistent coupled vibration-chemistry-vibration model. J. Thermophys. Heat Transf., 9(2), 219-226.

Koo, H., Raman, V., and Varghese, P. 2015. Direct numerical simulation of supersonic combustion with thermal nonequilibrium. Proc. Combust. Inst., 35, 2145-2153.

McBride, B.J., Zehe, M.J., and Gordon, S. 2002. NASA Glenn coefficients for calculating thermodynamic properties of individual species. NASA report TP-2002-2115 56. National Aeronautics and Space Administration, Cleveland, $\mathrm{OH}$.

Oran, E.S., and Boris, J.P. 2005. Numerical Simulation of Reactive Flow, Cambridge University Press, New York.

Oran, E.S., Weber, J.W., Stefaniw, E.I., Lefebvre, M.H., and Anderson, J.D. 1998. A numerical study of a two-dimensional $\mathrm{H}_{2}-\mathrm{O}_{2}$-Ar detonation using a detailed chemical reaction model. Combust. Flame., 113(1), 147-163. 
Panesi, M., Munafò, A., Magin, T.E., and Jaffe, R.L. 2014. Nonequilibrium shock-heated nitrogen flows using a rovibrational state-to-state method. Phys. Rev. E, 90(1), 013009.

Park, C. 1989. Assessment of two-temperature kinetic model for ionizing air. J. Thermophys. Heat Trans., 3(3), 233-244.

Ropp, D.L., and Shadid, J.N. 2009. Stability of operator splitting methods for systems with indefinite operators: Advection-diffusion-reaction systems. J. Comput. Phys., 228(9), 3508-3516.

Schott, G.L., and Kinsey, J.L. 1958. Kinetic studies of hydroxyl radicals in shock waves. II. Induction times in the hydrogen-oxygen reaction. J. Chem. Phys., 29(5), 1177-1182.

Shen, H., Liu, K.X., and Zhang, D.L. 2011. Three-dimensional simulation of detonation propagation in a rectangular duct by an improved CE/SE scheme. Chin. Phys. Lett., 28(12), 124705.

Shen, H., and Wen, C.Y. 2016. A characteristic space-time conservation element and solution element method for conservation laws. II. Multidimensional extension. J. Comput. Phys., 305, 775-792.

Shen, H., Wen, C.Y., Liu, K.X., and Zhang, D.L. 2015a. Robust high-order space-time conservative schemes for solving conservation laws on hybrid meshes. J. Comput. Phys., 281, 375-402.

Shen, H., Wen, C.Y., and Massimi, H.S. 2014. Application of CE/SE method to study hypersonic non-equilibrium flows over spheres. AIAA 2014-2509.

Shen, H., Wen, C.Y., and Zhang, D.L. 2015b. A characteristic space-time conservation element and solution element method for conservation laws. J. Comput. Phys., 288, 101-118.

Strehlow, R.A., and Engel, C.D. 1969. Transverse waves in detonations: II. Structure and spacing in $\mathrm{H}_{2}-\mathrm{O}_{2}, \mathrm{C}_{2} \mathrm{H}_{2}-\mathrm{O}_{2}, \mathrm{C}_{2} \mathrm{H}_{4}-\mathrm{O}_{2}$, and $\mathrm{CH}_{4}-\mathrm{O}_{2}$ systems. AIAA J., 7(3), 492-496.

Taylor, B.D., Kessler, D.A., Gamezo, V.N., and Oran, E.S. 2013a. Numerical simulations of hydrogen detonations with detailed chemical kinetics. Proc. Combust. Inst., 34, 2009-2016.

Taylor, B.D., Kessler, D.A., and Oran, E.S. 2013b. Estimates of vibrational nonequilibrium time scales in hydrogen-air detonation waves. Presented at the 24th International Colloquium on the Dynamics of Explosive and Reactive Systems, Taipei, Taiwan, July 28-August 2.

Voelkel, S.J., Raman, V., and Varghese, P. 2015. Non-equilibrium reaction rates in hydrogen combustion. Presented at the 25th International Colloquium on the Dynamics of Explosions and Reactive Systems (ICDERS), Leeds, UK, August 2-7. 